\title{
The role of guidelines and the patient's life-style in GPs' management of hypercholesterolaemia
}

\author{
Lars Backlund*1, Ylva Skånér ${ }^{1}$, Henry Montgomery², Johan Bring ${ }^{3}$ and Lars- \\ Erik Strender ${ }^{1}$
}

Address: ${ }^{1}$ Family Medicine Stockholm, Karolinska Institutet, Alfred Nobels allé 12, SE-141 83 Huddinge, Sweden, ${ }^{2}$ Department of Psychology, University of Stockholm, Sweden and 'Statisticon AB, Östra Ågatan 31, SE-753 22 Uppsala, Sweden

Email: Lars Backlund* - lars.backlund@klinvet.ki.se; Ylva Skånér - ylva.skaner@klinvet.ki.se; Henry Montgomery - hmy@psychology.su.se; Johan Bring - johan.bring@statisticon.se; Lars-Erik Strender - lars-erik.strender@klinvet.ki.se

* Corresponding author

Published: 09 March 2004

BMC Family Practice 2004, 5:3
Received: 26 September 2003

Accepted: 09 March 2004

This article is available from: http://www.biomedcentral.com/I47/-2296/5/3

(c) 2004 Backlund et al; licensee BioMed Central Ltd. This is an Open Access article: verbatim copying and redistribution of this article are permitted in all media for any purpose, provided this notice is preserved along with the article's original URL.

\begin{abstract}
Background: Recent Swedish and joint European guidelines on hyperlipidaemia stress the high coronary risk for patients with already established arterio-sclerotic disease (secondary prevention) or diabetes. For the remaining group, calculation of the ten-year risk for coronary events using the Framingham equation is suggested. There is evidence that use of and adherence to guidelines is incomplete and that tools for risk estimations are seldom used. Intuitive risk estimates are difficult and systematically biased. The purpose of the study was to examine how GPs use knowledge of guidelines in their decisions to recommend or not recommend a cholesterol-lowering drug and the reasons for their decisions.
\end{abstract}

Methods: Twenty GPs were exposed to six case vignettes presented on a computer. In the course of six screens, successively more information was added to the case. The doctors were instructed to think aloud while processing the cases (Think-Aloud Protocols) and finally to decide for or against drug treatment. After the six cases they were asked to describe how they usually reason when they meet patients with high cholesterol values (Free-Report Protocols). The two sets of protocols were coded for cause-effect relations that were supposed to reflect the doctors' knowledge of guidelines. The Think-Aloud Protocols were also searched for reasons for the decisions to prescribe or not to prescribe.

Results: According to the protocols, the GPs were well aware of the importance of previous coronary heart disease and diabetes in their decisions. On the other hand, only a few doctors mentioned other arterio-sclerotic diseases like stroke and peripheral artery disease as variables affecting their decisions. There were several instances when the doctors' decisions apparently deviated from their knowledge of the guidelines. The arguments for the decisions in these cases often concerned aspects of the patient's life-style like smoking or overweighteither as risk-increasing factors or as alternative strategies for intervention.

Conclusions: Coding verbal protocols for knowledge and for decision arguments seems to be a valuable tool for increasing our understanding of how guidelines are used in the on treatment of hypercholesterolaemia. By analysing arguments for treatment decisions it was often possible to understand why departures from the guidelines were made. While the need for decision support is obvious, the current guidelines may be too simple in some respects. 


\section{Background}

The decision to recommend or not to recommend drug treatment for individuals with high cholesterol values should depend on an evaluation of the individual's risk for future cardiovascular disease, primarily coronary heart disease (CHD). The European guidelines [1] emphasise the high risk for people with already established CHD or other arterio-sclerotic manifestations such as stroke, transient ischaemic attacks (TIA) or peripheral artery disease (PAD). For these so-called secondary prevention cases pharmacological treatment should generally be justified under the condition that life-style intervention has been tried for a sufficiently long time. The cut-off values for initiating treatment, which are the same as the goal values for treatment, are specified as $5.0 \mathrm{mmol} / \mathrm{L}$ for total cholesterol, and $3.0 \mathrm{mmol} / \mathrm{L}$ for low-density-proteins (LDL). The 1999 Swedish adaptation of the European guidelines [2] stresses the high coronary risk for individuals with type 2 diabetes and suggests that they should be screened and recommended treatment based on the same criteria as in the secondary prevention situation. For the remaining group of individuals with elevated cholesterol and/or elevated LDL levels who are not at high risk for other reasons (e.g. diagnosed as familial hypercholesterolaemia), the decision to recommend a drug or not should be based on a numerical estimate of the individual's absolute risk for a future coronary event. The European and Swedish guidelines recommend that the absolute risk for a coronary event within the next ten years should be calculated using the so-called Framingham equation [3]. If the 10 -year risk exceeds $20 \%$, or if it exceeds $20 \%$ when the time span is projected to the age of 60 , drug treatment should be considered if life-style interventions have not sufficed.

Due to the vast number of different guidelines of relevance for primary health care physicians, and the rapid changes in guidelines in the field of cholesterol treatment, we can assume that guidelines are not known in detail or easily retrievable by all doctors. Even if the guidelines were well known and/or easily retrievable, adherence to them could vary due to different opinions and values on the part of doctors. We can also assume that tools for risk estimates are not always available or used when indicated $[4,5]$. The consequences can be over- and undertreatment as compared with the guidelines, which has been shown by a review of medical records [6], and an inefficient use of quite costly cholesterol-lowering drugs. An increased understanding of how and why doctors' decisions on drug treatment deviate from guidelines may be useful in formulating and communicating guidelines and in teaching in general within preventive cardiology.

In the present work, we used the think-aloud technique [7] to find out how primary health care physicians make decisions about simulated patient cases with hypercholes- terolaemia in combination with other risk factors. In two previous papers that were based on the same think-aloud experiment, we investigated the validity of Think-Aloud Reports compared with rating data for reflecting the decision process over time [8], and we analysed the importance of different patient characteristics and risk factors regarding prescription decisions [9].

The aim of the present study was to investigate how general practitioners use their knowledge about the guidelines on hyperlipidaemia in their decisions on drug treatment, and what their arguments are for their decisions concerning cases with different recommended treatment according to the guidelines. We therefore analysed how the verbal protocols reflected the doctors' knowledge of guidelines with regard to the special status of individuals with CHD, other arterio-sclerotic manifestations, or diabetes. We used the coding method described by Axelrod [10] in his construction of "cognitive maps". Briefly, a cognitive map is a model of a person's belief system about a limited domain and consists of a number of cause-effect relations. The analysis of the verbal protocols according to their cause-effect structure proved to be more suitable than more complex coding schemes because of the quite simple conceptual structure of our data.

As a separate analysis, we examined the evidence from the verbal protocols regarding arguments for decisions to prescribe or not to prescribe for different patient cases. In particular, the analysis of arguments can be interesting when the participant seems to be aware of a certain aspect of the guidelines, for example that the presence of $\mathrm{CHD}$ is a strong indication for drug treatment for an elevated cholesterol value, but still decides not to treat a patient with these characteristics. Two kinds of verbal protocols were used. The protocols from processing and deciding upon the simulated cases (Think-Aloud Protocols) were complemented by protocols from a task in which the doctors were asked to describe how they usually reason in their practice when drug prescription for elevated cholesterol values may be indicated (Free-Report Protocols). In this way the doctors not only got "a second chance" to express their knowledge and decision rules concerning the risk factors presented with the cases, but also to state their rules and knowledge regarding diseases that were not represented in any of our case vignettes (e.g., stroke). Moreover, we could examine what factors other than the traditional risk factors, such as those presented in the case vignettes, seemed to influence decisions on drug treatment. For example, we could expect some participants to talk about the patient's compliance, side effects of drugs, drug costs and so on as factors influencing the decision to prescribe or not to prescribe for individuals with high cholesterol values. These latter protocols were therefore examined not only concerning evidence of knowledge of 
guidelines but also concerning all variables affecting the decision to prescribe or not to prescribe a drug for hypercholesterolaemia.

\section{Methods \\ Participants}

Ten male and 10 female GPs aged 34 to 60 years (mean = 48.3 ) participated. They were all specialists in family medicine and had practised between one and 22 years (mean $=11.4$ ) as such. A total of 36 doctors from the southern Stockholm area were contacted by telephone and twentyfour agreed to participate. The remaining 12 doctors (five males and seven females with mean age 47.0 years) almost invariably reported a lack of time as their reason for not participating. The four who later declined to participate (usually by fax) were not asked for their reasons.

\section{Design}

The order of the cases was the same for all participants. Cases with "Yes" and "No" decisions as the recommended treatment according to the guidelines were mixed as evenly as possible. In order to examine the validity of think-aloud protocols, 10 of the participants were randomly assigned to a condition where, in addition to thinking aloud, they also rated their willingness to prescribe a drug at regular intervals during each case. It was shown in an earlier study that rating data and think-aloud data corresponded closely concerning how they reflected the directionality of the decision process, and were hence considered to mutually validate each other [8]. In the present paper the protocols from the two experimental groups were not separated.

\section{Cases}

Six authentic clinical cases with a cholesterol level of at least $5.5 \mathrm{mmol} / \mathrm{L}$ were selected to represent secondary pre- vention, diabetes and primary prevention cases with a spread of the 10 -year coronary risks. The central information describing the six different cases, as well as the recommended treatment according to Swedish guidelines are described under Results (Table 4). The treatment recommendations are based on the assumption that patients with CHD, other arterio-sclerotic manifestations or diabetes should be recommended drug treatment, while patients who are free from these diseases should be recommended treatment if their estimated 10 -year risk for a coronary event exceeds $20 \%$. All patients were assumed to have tried for a reasonable amount of time to normalise their cholesterol values with diet and exercise.

The different kinds of clinical information were presented on six successive screens, and the information was divided time-wise in the same way for all six cases. The order in which this information was presented was arranged so as to be as realistic as possible in relation to clinical practice (including how patient cases are described in written referrals to other clinics and in clinical conferences and tutoring). Table 1 shows case IS as it was presented to participants in the study. All previously shown information about a case was repeated on the later screens to reduce and control for memory effects. This part of the text was placed at the top of the screen and was a different colour.

\section{Procedure}

The study was carried out between August and December 2000. The details of the procedure have been described previously [8]. In the course of six screens, more clinical information was gradually added to the case. The participants were instructed to voice aloud all their thoughts about the case, and were told that each case would end with the question as to whether or not they would prescribe a drug for this patient.

Table I: Example of a Case (IS)

\begin{tabular}{|c|c|}
\hline Screen & Information \\
\hline 1 & 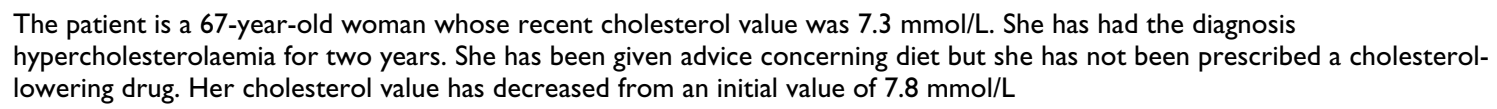 \\
\hline 2 & $\begin{array}{l}\text { The patient has been on medication for hypertension for } 10 \text { years (Seloken } \mathrm{ZOC} * 50 \mathrm{mg} \text { and Plendil** } 5 \mathrm{mg} \text { ). She is now on a } \\
\text { visit to check her blood pressure and hypercholesterolaemia. }\end{array}$ \\
\hline 3 & $\begin{array}{l}\text { The patient has no other known diseases apart from osteoarthritis of her knees. Her mother suffered from hypertension and } \\
\text { reached the age of } 84 \text { years. }\end{array}$ \\
\hline 4 & $\begin{array}{l}\text { The patient is a non-smoker. She very seldom drinks alcohol. She does not exercise on a regular basis but she is fond of taking } \\
\text { walks. }\end{array}$ \\
\hline 5 & $\begin{array}{l}\text { Physical examination: Good general condition. A few kilograms overweight. Blood pressure } 145 / 75 \text {. Heart and lung auscultation } \\
\text { normal. }\end{array}$ \\
\hline 6 & $\begin{array}{l}\text { Laboratory values: Total cholesterol } 7.3 \mathrm{mmol} / \mathrm{L} \text {. LDL } 5.4 \mathrm{mmol} / \mathrm{L} \text {. HDL } 1.2 \mathrm{mmol} / \mathrm{L} \text {. Triglycerides } \mathrm{I} .6 \mathrm{mmol} / \mathrm{L} \text {. TSH, creatinine } \\
\text { and liver-function tests were normal. }\end{array}$ \\
\hline 7 & $\begin{array}{l}\text { Would you prescribe a cholesterol-lowering drug for this patient? } \\
\text { Yes } \square \text { No } \square\end{array}$ \\
\hline
\end{tabular}


After the six cases the participants were asked to describe in their own words how they usually reason when confronted with patients for whom drug treatment for high cholesterol values could be considered (Free Reports).

The study was approved by the local ethics committee.

\section{Response measures and coding of data \\ Decisions}

Each case ended with a screen (screen seven) with the following question: "Would you prescribe a cholesterol-lowering drug for this patient?" The participant responded by clicking on one of two response alternatives, "Yes" or "No".

\section{Think-Aloud Protocols}

The sessions were tape-recorded. A secretary then transcribed the recorded sessions into a written, word-byword format. The protocols were segmented into statements. For the purpose of the present paper, the ThinkAloud Protocols were searched for two aspects, arguments concerning the decision to prescribe or not to prescribe, and (as described under Free-Report Protocols below) certain cause-effect relations. The criteria for an argument were that it should be voiced in connection with a statement indicating the decision (or a consideration of the decision) and that it should be interpreted by the coder as providing increased understanding of the decision. The following are examples of such statements. "I say no because I would like her diabetes to be under better control and her weight reduced or for her to get more physical exercise." (Diabetic case GM, Participant No. 11); "I think his cholesterol value is so close to normal and the diagnosis of angina pectoris seems to be unclear, so I would hesitate to prescribe" (CHD case AR, Participant No. 4).

In the original coding, the statements were also categorised with respect to the cognitive content, their directionality in relation to the decision (speaking for or against drug treatment), and finally which information about the case the statement referred to. These aspects of the coding are described in previous papers [8,9] and are not used in the present article.

\section{Free-Report Protocols}

The protocols from the Free Reports were segmented into statements. The set of statements was thereafter analysed for cause-effect relations according to the method described by Axelrod [10]. (As the Free Reports were not related to the different cases, coding for decision arguments was not considered relevant). Both causes and effects are variables in the sense that they can have different values. In the present context, cause variables are mainly patient-describing variables, e.g. age, presence or absence of different medical conditions, and laboratory values. A cause can be a single variable or a combination of other cause variables, e.g. elevated cholesterol in combination with hypertension.

The coding resulted in 34 different single cause variables and 39 different combinations of two or three of the same cause variables, i.e. altogether 73 different causes affecting an effect variable. We originally coded 23 different effect variables. The result was a number of cause-effect relations that were not directly relevant to the task in question (e.g. increased alcohol intake causing an increase in Triglycerides levels). We present only cause-effect relations that include three effect variables that were judged to be pertinent to the task the doctors were instructed to carry out.

\section{Usability}

This effect variable indicates that a certain type of information (cause) is useful for the decision situation without referring to the probability for a prescription. Examples of expressions by the participants (with the cause expression within parentheses) were: " It's of little use...(to refer to a specialist)"; " I often look at ...(risk tables)".

\section{Probability}

A general influence on the probability for drug prescription is exemplified by the following statements (causes within parenthesis): Participant No. 13- "(The patient's own opinion)...affects my willingness to prescribe"; Participant No. 8- "(If the patient is very old...and considering the cost of drugs) you should really think twice before prescribing a drug ".

\section{Implication}

If, on the other hand, the effect seemed to be a definite or almost definite prescription given the cause variable(s) at hand, the effect variable was termed "Implication". Examples of statements coded in this way were (causes within parentheses): Participant No. 1- "I treat patients with (ischaemic heart disease)"; Participant No. 4- "(If the patient is young, has a level below 8 , and no other diseases) I wouldn't treat it".

We did not distinguish between positive and negative directions (or other possible kinds of relations as specified by Axelrod [10]) in the cause-effect relations. The relation between cause and effect can be positive in the sense that an increased value, or presence, of the cause variable leads to an increased value in the effect variable. For example, increased age of the patient leads to a higher probability of drug prescription. Correspondingly, a negative relation implies that an increased value of the cause variable leads to a decreased value of the effect variable. In some instances, the direction could be understood tacitly and in other instances it could not be determined (e.g. when a participant stated that smoking affected the probability 
Table 2: The frequencies of different cause-effect combinations in the Free-Report Protocols.

\begin{tabular}{|c|c|c|c|c|c|}
\hline \multirow[t]{2}{*}{ Cause variable } & \multicolumn{4}{|c|}{ Effect variable } & \multirow[t]{2}{*}{ No. of Drs } \\
\hline & Usability & Probability & Impli cation & Total & \\
\hline Cholesterol/ Lipid Level & 4 & 11 & 19 & 34 & 12 \\
\hline CHD & 1 & 8 & 12 & 21 & 7 \\
\hline Diabetes & 0 & 11 & 9 & 20 & 15 \\
\hline Family History & 0 & 10 & 7 & 17 & 14 \\
\hline Age & I & 12 & 2 & 14 & 7 \\
\hline Smoking & 0 & 11 & 0 & 11 & 11 \\
\hline Cardio-Vascular Disease & 0 & 3 & 8 & 11 & 8 \\
\hline Risk Level/Risk Chart & 5 & 4 & 1 & 10 & 9 \\
\hline Life Style Change/Factors & 0 & 2 & 7 & 9 & 5 \\
\hline LDL & 1 & 7 & 1 & 9 & 6 \\
\hline Hypertension & 0 & 6 & 2 & 8 & 6 \\
\hline Patient's Attitude & 0 & 7 & I & 8 & 6 \\
\hline PAD & 0 & 3 & 4 & 7 & 4 \\
\hline HDL & 0 & 6 & 1 & 7 & 4 \\
\hline Stroke/TIA & I & 3 & 2 & 6 & 4 \\
\hline Overweight & 0 & 6 & 0 & 6 & 6 \\
\hline Side Effects of Drugs & 0 & 3 & 0 & 3 & 3 \\
\hline Exercise & 0 & 3 & 0 & 3 & 3 \\
\hline Patient's Sex & 0 & 3 & 0 & 3 & 2 \\
\hline Alcohol Consumption & 0 & 2 & 0 & 2 & 2 \\
\hline Cost/Cost Benefit & I & 1 & 0 & 2 & 2 \\
\hline Local Organisation & 2 & 0 & 0 & 2 & 2 \\
\hline Dr-Patient Relation & 2 & 0 & 0 & 2 & 2 \\
\hline
\end{tabular}

for drug prescription but not whether the impact was positive or negative). Due to a fairly large number of such implicit or undeterminable directions, we did not distinguish between different kinds of relations between causes and effects in the data presentation. In a few cases when an effect was also a cause of a different effect variable (i.e. a chain of cause-effect relations), we analysed this as two separate cause-effect relations.

\section{Results}

Our main purpose was to investigate how the doctors use their guidelines knowledge and what arguments they give for their decisions on drug treatment. First, however, we will give an overview of the Free-Report Protocols. Thereafter, we will combine the three different kinds of data (decisions, cause-effects relations and arguments) to try to elucidate the decisions on the different cases.

\section{Contents of the Free-Report Protocols Reliability}

Two of the authors (LB and YS) coded 50 randomly selected statements from the Free-Report Protocols independently of each other. Before that, and in order to get a common understanding of how the variables were defined, an initial coding of another 10 randomly selected statements was performed and, when necessary, differ- ences in opinions were discussed and resolved. The cause and effect variables in the presentation of the final results (Table 2) were used. For 39 (78\%) of the statements there was complete agreement as to the cause variable or variables. The same rate of agreement $(78 \%)$ was found for effect variables. There were 31 statements $(62 \%)$ in which there was agreement regarding both cause(s) and effect.

The total number of coded relations with the three effect variables Usability, Probability and Implication was 200, or on average 10.0 per doctor. The most frequent effect variable was Probability (mean 6.1), followed by Implication (mean 2.4) and Usability (mean 1.5). The vast majority $(82 \%)$ of the coded relations were restricted to one cause variable, $14.5 \%$ to two and $3.5 \%$ to three cause variables in combination. It can be noted that four of the seven relations with three causes stemmed from one participant. Nine of the 20 participants gave no relations with more than one cause variable. There was considerable variation in the length of the Free Report Protocols of the different participants. The number of words varied between 158 and 773, and the number of cause-effect relations varied between two and 22 .

Table 2 shows the number of expressed relations containing different cause variables related to the three different 
Table 3: The number of doctors with protocols containing cause variables relevant for secondary prevention and diabetes.

\begin{tabular}{|c|c|c|c|c|c|c|}
\hline \multirow[t]{2}{*}{ Cause variable } & \multicolumn{2}{|c|}{ Think aloud (TA) } & \multicolumn{2}{|c|}{ Free report } & \multicolumn{2}{|c|}{ TA or Free report } \\
\hline & Probability & Implication & Probability & Implication & Probability & Impli cation \\
\hline $\mathrm{CHD}$ & 5 & 12 & I & 6 & 5 & 14 \\
\hline CHD or Cardiovascular-, or Heart Disease & 5 & 14 & 3 & 11 & 3 & 17 \\
\hline Diabetes & 10 & 9 & 6 & 9 & 8 & 11 \\
\hline Stroke/TIA & 0 & 0 & I & 3 & I & 3 \\
\hline Stroke/TIA or Cardio/Cerebro-vascular Disease & 2 & 2 & 4 & 5 & 3 & 7 \\
\hline Peripheral Artery Disease & 0 & 0 & 0 & 4 & 0 & 4 \\
\hline $\begin{array}{l}\text { Peripheral Artery Disease or Cardiovascular } \\
\text { Disease }\end{array}$ & 2 & 1 & I & 7 & 0 & 8 \\
\hline
\end{tabular}

effect variables. The frequencies include instances when the cause variable was expressed either alone or in combination with one or two other cause variables. The last column shows the number of doctors out of 20 who had the different cause variables included in their cause-effect relations. For the sake of clarity, some cause variables have been aggregated, e.g. Patient's Attitude (includes Motivation and Opinion among other original cause variables) and Cardio-Vascular Disease (includes Heart Disease and Cerebro-Vascular Disease). A specific combination of cause(s) and effect was not coded more than once for every participant, but it was possible for a participant to have both Probability and Implication, for example, related to the same cause. The cause variables are ordered from top to bottom with regard to the total frequencies of relations coded. Cause variables with a frequency of at least two are included in Table 2. Cholesterol level was the most frequent cause variable, followed by CHD, Diabetes, Family History and Age in decreasing frequency. If we restrict the analysis to single cause variables, Diabetes was the most common variable, followed by Smoking, Age and the combined variable Risk Level/Risk Chart. Diabetes was also the cause variable with the highest probability of being represented in a doctor's protocol, followed by Family History. The most frequent single cause-effect relation was Smoking affecting Probability, which was present for 12 of the 20 participants. As single cause variables, life-style factors (like Smoking and Overweight) and Family History caused changes in the probability of a drug prescription, but never to the extent that it was coded as implying drug prescription. The variable Life-Style Change/Life-Style Factors was related to Implication when combined with other causes, but restricted to Probability when it was a single cause variable. There were a few relations with cause variables outside the traditional risk factors. The most common was the Patient's Attitude with Probability as effect for seven participants and Implication for one participant. Other such cause variables were Side Effects of Drugs, Cost-Benefit considerations and local resources and routines ("Local Organisation"). The variables that most often affected Usability were Risk Level/Risk Chart and Cholesterol/Lipid Level.

\section{Evidence of knowledge related to guidelines}

The cause variables of interest for this part of the analysis were Diabetes, CHD, Stroke/TIA, and PAD. The latter three variables were also analysed as subgroups of the more general terms Cardio-Vascular Disease (relevant for all three cause variables), Heart Disease (relevant for CHD) and Cerebro-Vascular Disease (relevant for Stroke/ TIA). Table 3 shows the number of doctors who expressed relations in the Think-Aloud Protocols and the FreeReport Protocols containing these different cause variables (alone or in combination with other cause variables) and the effect variables Probability and Implication. There were only two instances when Usability was related to these cause variables, one for Stroke and one for Heart Disease, and these figures are not included in Table 3. If a protocol from a participant contained both Probability and Implication for a certain cause variable, only Implications was counted.

Fourteen of the 20 doctors indicated that CHD alone, or in combination with other patient variables, should lead to drug prescription (Implication), and this was the case for a few more of the doctors if the cause variable was generalised to Cardio-Vascular Disease or Heart Disease. For Diabetes, the figure was 11 out of 20 . If we include Probability, practically all the doctors revealed having this knowledge regarding Diabetes and CHD in one or two of the protocols. It also seems that most of the knowledge concerning CHD and Diabetes was manifested in the Think-Aloud Protocols and that little was added in this respect by also taking the Free-Report Protocols into account. Few doctors expressed the association regarding Stroke/TIA and PAD even if the more general terms cardio-vascular and cerebro-vascular diseases were included. This is to be expected, as CHD and diabetes were repre- 
Table 4: Description of the six cases and the percentage of doctors who decided to prescribe for each case.

\begin{tabular}{|c|c|c|c|c|c|}
\hline Case & $\begin{array}{c}\text { Cholesterol } \\
\text { value }(\mathrm{mmol} / \mathrm{L})\end{array}$ & Additional risk factors & $\begin{array}{l}\text { Recommended } \\
\text { management }\end{array}$ & $\begin{array}{l}\text { Estimated risk in } \\
\text { percentage by chart }\end{array}$ & $\begin{array}{l}\text { Percentage of doctors } \\
\text { who decided to prescribe }\end{array}$ \\
\hline $\begin{array}{l}\text { AR } \\
\text { (male, 56) }\end{array}$ & 5.9 & CHD, Overweight & Yes (CHD) & $*$ & 85 \\
\hline $\begin{array}{l}\text { GM } \\
\text { (female, 53) }\end{array}$ & 5.9 & Diabetes, slight Family History & Yes (diabetes) & 10-20 (moderate) & 50 \\
\hline $\begin{array}{l}\text { TW } \\
\text { (male, 67) }\end{array}$ & 6.0 & Hypertension, Smoking & Yes (risk above $20 \%$ ) & $20-40 \%$ & 35 \\
\hline $\begin{array}{l}\text { IS } \\
\text { (female, 67) }\end{array}$ & 7.3 & Hypertension, slight Family History & No (risk below $20 \%$ ) & 10-20 (moderate) & 60 \\
\hline $\begin{array}{l}\text { PU } \\
\text { (female, 4I) }\end{array}$ & 7.2 & Strong Family History & $\begin{array}{l}\text { No (risk below 20\%) } \\
\text { ** }\end{array}$ & $10-20 * *$ & 70 \\
\hline $\begin{array}{l}\text { SH } \\
\text { (female, 5I) }\end{array}$ & 6.5 & No additional risk factors & No (risk below $20 \%$ ) & 5-10 (mild) & 0 \\
\hline
\end{tabular}

* Risk estimation is not considered relevant in the presence of CHD ** Family history or Familial hypercholesterolaemia are not directly included in the charts

sented in the case vignettes, whereas no patient had a cerebro-vascular disease or PAD, nor were these diseases explicitly denied in the case descriptions.

\section{Decisions and arguments}

Table 4 shows the percentage of doctors who decided to prescribe for the different patient cases along with the central characteristics of the cases. The order of the cases in Table 4 follows the presentation below, while the order of occurrence for the participants was IS-GM-TW-SH-AR-PU.

\section{CHD case}

For the CHD case (AR), the presence of angina pectoris should be a strong indication for drug therapy. Of the 20 doctors, 17 decided to recommend a drug. The number of doctors who expressed the effect variable Implication regarding CHD or Cardio-Vascular Disease was 17 as well. There was no overlap between the group of three who decided to refrain from treatment and the three who did not express this knowledge. From the Think-Aloud Protocols we found that one of the three doctors who refrained from treatment questioned the diagnosis of angina pectoris and two suggested that weight reduction should be further encouraged before starting drug treatment.

\section{Diabetic case}

For the diabetic case (GM), 10 of the 20 doctors suggested drug prescription. In the "Yes" group six out of 10 verbalised Implication. In the "No" group the corresponding number was five. If we also incorporate Probability, the numbers were $10 / 10$ in the Yes- group and 9/10 in the "No" group. In other words, almost every doctor stated that diabetes in combination with hypercholesterolaemia increases the reason to prescribe a cholesterol-lowering drug, and there was no clear difference between those who finally decided to recommend a drug and those with the opposite decision in this respect.

Using the Think-Aloud Protocols we can examine the reasons given by the 10 doctors who chose to refrain from treatment. Three doctors expressed an inclination to prescribe a drug but said at the same time that they preferred to wait and see. Two doctors said that they wanted to await further change in life-style. Another three doctors seemed to have identified arguments against treatment (lack of other diseases and risk factors, a mild form of diabetes, marginally elevated cholesterol). Two of the 10 doctors who decided to refrain from treatment gave no clear motivation in their verbal protocols (e.g. Dr 2: " I don't think we have anything to gain").

\section{Primary prevention cases}

The high-risk case (TW) is the first of the primary prevention cases to be examined here. The presence of smoking and hypertension in this 67-year-old male with a cholesterol value of $6.0 \mathrm{mmol} / \mathrm{L}$ gives a 10 -year risk in the range of $20-40 \%$ when the risk chart is applied, which would justify pharmacological treatment. However, this was the choice of only $35 \%$ of the participants. The following are examples of arguments expressed by 13 of the doctors who decided not to prescribe. Six doctors said that the patient should stop smoking first, two that physical exercise should be tried first. One doctor seemed to use the moderate cholesterol elevation as an argument for not recommending a drug. For the remaining four non-prescribers no clear arguments for their decision could be found in the protocols. Among the seven who chose to prescribe, four seem to have used the patient's inability to change his life-style as their motivation and the other three mentioned multiple risk factors. In other words, life-style factors seemed to have been important in making the 
decision, but as an argument both for prescribing and for not prescribing.

Case IS represents a patient with moderate risk (10-20\%), which would ordinarily not motivate drug treatment. However, 12 of the 20 participants $(60 \%)$ chose to prescribe. Ten of the 12 prescribers for Case IS gave hypertension as an argument and four of these also mentioned the degree of cholesterol- and/or LDL elevation as well. One of the four also included the information about family history. One of the doctors who chose to prescribe gave no argument for his/her decision and one merely stated that life-style intervention had not succeeded. For the eight non-prescribers, we could not find any argument for their decision in four of the verbal protocols, whereas two doctors stressed the small number risk factors, one that the patient was female and had a high HDL, and one that the patient was a non-smoker and had a mother who had reached an advanced age.

Case PU describes a young female with no risk factors other than a fairly high cholesterol/LDL and a severe family history of coronary heart disease. The case may qualify as "probable familial hypercholesterolaemia" [11]. A simple application of the risk chart would give a mild risk (5$10 \%)$ or, more correctly, a moderate risk (10-20\%) if the time span were projected to the age of 60 years. However, the severe family history should be taken into account, which would make drug treatment reasonable. The majority ( 14 of 20 or $70 \%$ ) chose to prescribe. All the prescribers gave family history as an argument for drug treatment and four seemed to have considered the diagnosis familial hyperlipidaemia. Of the six non-prescribers, three gave no apparent argument for their decisions; two appeared to have used the patient's young age and long expected treatment time as an argument against prescription. The sixth non-prescriber apparently considered a number of risklowering factors (female sex, normal weight, non-smoking and physically active) as arguments against treatment.

Case SH represents a low-risk case (5-10\%) and all doctors chose not to prescribe. As arguments for their decisions, 11 doctors used terms like "no risk factor other than elevated cholesterol", four referred to the absence of other important diseases - diabetes, cardiovascular disease and hypertension, one doctor stated that the case did not concern secondary prevention, whereas another four doctors gave no clear reasons for their decision.

\section{Discussion}

Our purpose was to study the relation between the doctors' knowledge of guidelines and their decisions on patient cases. The concept of guidelines and evidencebased medicine implies, simply speaking, that patients should receive uniform treatment regardless of which doc- tor they happen to meet. The degree of consensus among the 20 doctors regarding the decisions varied considerably for the six cases - from a complete consensus on case SH to maximum possible variability in "Yes" and "No "decisions for case GM. At first glance, this might seem problematic as regards the quality of care for this patient group. However, when we combine the decisions with evidence of guideline knowledge and arguments for the decisions, the management of the different cases seems rather understandable. The diabetes case with as many "Yes" decisions as "No" decisions had only a marginally increased cholesterol level. For both of the cases that could be solved by applying a rule from the guidelines (i.e. the CHD case, AR, and the diabetes case, GM), there were several instances where a doctor seemed to know the content of the guidelines but nevertheless decided to depart from it. There seemed to be two types of arguments for the decision not to follow the guidelines strictly. One was the absence of risk factors other than hypercholesterolaemia. The other was giving priority to a life-style change rather than to drug treatment. For example, the patient should try to quit smoking or lose weight rather than start lipid-lowering medication. It is noteworthy that life-style factors could be used as an argument for decisions in two different ways. A modification of life-style could be seen as a means of reducing the total risk and thus as an alternative to drug treatment as previously discussed, or life-style could be seen as a risk factor per se. This is illustrated by Case TW: some participants viewed smoking cessation as an alternative action to drug treatment, while others used smoking as risk-increasing factor and therefore as an argument for drug treatment.

The decisions on the high-risk patient (TW) with several risk factors suggest a tendency to underestimate total risk for patients with many simultaneous risk factors (only seven of the 20 participants suggested drug prescription whereas the risk chart puts him in a high-risk category). This is in line with a previous study [4] where the task was to make numerical risk estimates of patient cases with elevated cholesterol values and different patterns of other risk factors: there was a tendency to underestimate risk, especially so for high-risk patients. However, other studies have shown a tendency for doctors to underestimate the absolute risk for coronary events when they make intuitive judgements [12,13].

For case IS with a moderate risk (implying no drug treatment) there was no evidence of under-treatment in relation to risk estimates and guidelines, as the majority $(60 \%)$ decided to prescribe.

The analysis of the Think-Aloud Protocols and the FreeReport Protocols implied a high degree of knowledge concerning two central aspects of the guidelines, the special 
treatment indications for patients with CHD and patients with diabetes. On the other hand, only a minority of the doctors stated that Stroke/TIA or peripheral artery disease were important as indications for drug treatment in the same sense as CHD and diabetes. There can be two reasons for this. Our choice of patient cases could be relevant. None of the cases had these diseases nor were the conditions denied in the descriptions, and consequently the doctors were not reminded of their existence. Secondly, the message that these arterio-sclerotic manifestations should be as important criteria for drug prescription as CHD and diabetes was quite new when the study was conducted (the guidelines were published in 1999 and the study was carried out in 2000), and it is possible that the doctors were not completely cognizant of this part of the guidelines.

Quite a few factors other than the traditional risk factors were found as cause variables in the Free-Report Protocols. The two most frequent variables both affected Probability, and they were the Patient's Attitude, including Willingness to Take Drugs, and Side-Effects of Drugs. It can be noted that in spite of the recurrent debate concerning high drug costs for cholesterol treatment, there were only two doctors who mentioned drug cost or cost-benefit considerations as factors influencing the decision or decision situation.

The Free-Report Protocols coded as cause-effect relations were supposed to reflect factors of importance regarding the decision to prescribe or not to prescribe a lipid-lowering drug. A comparison with the Think-Aloud Protocols from when the cases were processed, which is described in a separate paper [9], can lend some support to the validity in this respect. The different factors describing the patients were compared with regard to how often they were evaluated in a positive or negative way in relation to drug prescription. The six most important factors, in descending frequencies, were Cholesterol, Smoking, Hypertension, Family History, CHD and Diabetes. Approximately the same set of important variables was found in the FreeReport Protocols (see Table 2), in descending frequencies: Cholesterol, CHD, Diabetes, Family History, Age and Smoking.

The guidelines may be too simple in the sense that the same cut-off values for total cholesterol and LDL are used for all patients with established arterio-sclerotic disease or diabetes, regardless of the levels of their other risk factors. Our diabetes case demonstrated that an application of the risk chart suggests a different decision than simply applying the rule that diabetics should be regarded as on a par with secondary prevention cases. It can be noted, however, that the guidelines that were just recently published in Sweden [14] indicate that the threshold for patients at very high risk should be lower than $5.0 \mathrm{mmol} / \mathrm{L}$. Furthermore, the validity of the Framingham equation can be questioned on at least two grounds. One concerns the risk factors included. A severe family history of CHD should increase the risk substantially, but this risk factor does not enter into the Framingham formula. The other concerns the generalisability of the Framingham equation to new populations. There is agreement that the Framingham equation overestimates risk for populations with a low incidence of CHD [15], but recent results also indicate over-estimations for northern European samples [16].

Decisions on drug treatment or not in the primary prevention situation could be based on a formal or intuitive risk estimate. Intuitive risk estimates seem to be systematically biased $[4,12,13]$. There is also evidence that tools for risk estimates are seldom used [17], or little known [18] by different groups of European GPs. The data from the present study indicate that most doctors make the distinction between primary and secondary prevention in their decisions, but that recent modifications of the guidelines, e.g. the importance of previous stroke, is less well implemented. In the implementation of guidelines on preventive cardiology and lipid treatment there is a need not only to improve the availability and usability of guidelines, but also to make clear the distinction between primary and secondary prevention in a broader sense and to update the guidelines with risk assessment tools based on relevant risk data.

\section{Limitations of the study}

Conclusions concerning the participants' knowledge of certain aspects of the guidelines should be viewed in light of the method used. The validity of verbal protocols, including the coding procedure, can be questioned on several grounds. We did not interview the doctors directly about the guidelines but rather asked them to describe freely how they usually reason in connection with the decision situation at hand. It is possible that factors such as communication style, concentration, memory and tiredness might lead to an underestimation of knowledge on the part of the doctors. On the other hand, Free-Report Protocols and Think- Aloud Protocols have the advantage as compared to other information acquisition methods, involving more asking specific questions to the doctors, that they are minimally affected by the investigator's expectations. The Free-Report Protocols may have been affected by the decisions made in the previous cases as the participants may have strived to be consistent. Also, as discussed earlier, conditions that were not mentioned in the case descriptions (like Stroke/TAI) probably had a smaller chance to be mentioned in the Free-Report Protocols.

Another aspect of the validity problem concerns the congruence between medical knowledge and responses to 
simulated patient cases. Results from a think-aloud study by Kaufman and coworkers [19] regarding cases of hypercholesterolaemia and coronary heart disease suggest that medical knowledge is not always used adequately in decisions on patient cases.

The coding procedure may also be questioned, as the distinction between the effect variables Probability and Implication was not always self-evident, even if the interjudge reliability could be considered as reasonable (78\%). Another problem was that participants used different levels of abstraction when talking about diseases. For example, we could not know how many of the diseases in question, Stroke/TIA, CHD and PAD, were tacitly understood and encompassed by the expression "Cardio-Vascular Diseases". Finally, the coding of Think-Aloud Protocols for decision arguments was made by only one of the authors and the reliability has not been determined.

The purpose of the study was restricted to examining decisions without access to guidelines. A comparison of information processing and decisions with and without guidelines would be interesting. Patel and co-workers [20] used think-aloud technique to contrast performance with and without access to guidelines. They found that guidelines seemed to be used differently and serve different purposes by physicians at different level of expertise, a result that should have implications for the design of guidelines.

The number of cases in this study was small, and conclusions concerning different kinds of patient cases must be drawn with caution. Both the diabetic case and the CHD case had few other risk factors and the doctors' departure from a strict application of the guidelines should be viewed in this light. A better assessment of their adherence to guidelines with secondary prevention cases and diabetes would have required a larger set of cases with different degrees of cholesterol levels and other risk factors.

The analyses were done at a group level and no evaluation was carried out concerning how consistent individual doctors were across cases.

The GPs in the study were recruited from a limited geographical area, the southern part of Stockholm, and they may not be representative of Swedish GPs as regards knowledge in the medical field or the availability of local decision supports. Thus, it is possible that another sample of doctors would be quite different in their prescription decisions as well as the importance attributed to different patient characteristics.

\section{Conclusions}

One conclusion from the present study is that coding of verbal protocols for knowledge and for decision argu- ments is a valuable tool for increasing our understanding of how guidelines are used in decisions on realistic patient cases. Another conclusion is that it is problematic to use existing guidelines on lipid management and associated risk charts as a simple means to evaluate the quality of care for patients with hypercholesterolaemia.

\section{Competing interests}

None declared.

\section{Authors' contributions}

All authors participated in the design of the study. LB carried out the data collection. LB performed the coding of protocols, performed the rest of the data analyses and drafted the manuscript. All authors participated in the discussion of the drafts. All authors read and approved the final manuscript.

\section{Acknowledgements}

We thank the participating GPs. The study was supported by grants from the Stockholm County Council and the Swedish Heart Lung Foundation

\section{References}

I. Wood D, DeBacker G, Faegerman O, Graham I, Mancia G, Pyörälä K: Prevention of coronary heart disease in clinical practice. Summary of recommendations of the second joint task force of European and other societies on coronary heart prevention. Eur $H$ J 1998, 80: I- 10.

2. Treatment of hyperlipidaemia- recommendations. (Behandling av hyperlipidemi-rekommendationer.). Information från Läkemedelsverket 1999, 7:I5-21.

3. Anderson KM, Wilson PWF, Odell PM, Kannel WB: An updated coronary risk profile. A statement for health professionals. Circulation 1991, 83:356-362.

4. Backlund L, Bring J, Strender L-E: How accurately do general practitioners and students estimate coronary risk in hypercholesterolaemic patients. Primary Health Care Research and Development 2004, 5:145-152.

5. Ramachandran S, Labib $\mathrm{MH}$ : Hyperlipidaemia and primary prevention of coronary heart disease: are the right patients being treated? J Cardiovasc Risk 2000, 7:245-249.

6. Abookire SA, Karson AS, Fiskio J, Bates DW: Use and monitoring of "statin" lipid-lowering drugs compared with guidelines. Arch Intern Med 2001, I 61 1:2626-2627.

7. Ericsson KA, Simon HA: Protocol Analysis: Verbal reports as data Cambridge, MA, US: The MIT Press; 1984.

8. Backlund L, Skånér Y, Montgomery H, Bring J, Strender L-E: Doctors' decision processes in a drug prescription task: The validity of rating scales and verbal reports. Organ Behav Hum Decis Proces 2003, 91 : 108-II7.

9. Backlund L, Skånér Y, Montgomery H, Bring J, Strender L-E: GPs' decisions on drug treatment for patients with high cholesterol values: A think-aloud study (manuscript). .

10. Axelrod R: The analysis of cognitive maps. In Structure of Decision Edited by: Axelrod R. Princeton: Princeton Univ Press; 1976:55-73.

II. Neil HAW, Hammond T, Huxley R, Matthews DR, Humphries SE: Extent of underdiagnosis of familial hypercholesterolaemia in routine practice: prospective registry study. $\mathrm{Br}$ Med J 2000, $321: 148$.

12. Friedman PD, Brett AS, Mayo-Smith MF: Differences in generalists' and cardiologists' perceptions of cardiovascular risk and the outcomes of preventive therapy in cardiovascular disease. Ann Intern Med 1996, I 24:4|4-42I.

13. Pignone M, Phillips CJ, Elasy TA, Fernandez A: Physicians' ability to predict the risk of coronary heart disease. BMC Health Services Research 2003, 3: I3.

14. Treatment with lipid-lowering drugs in the prevention of cardio-vascular diseases. (Behandling med lipidsänkande 
läkemedel vid prevention av hjärt-kärlsjukdomar.). Information från Läkemedelsverket 2003, 14:15-21.

15. Marrugat J, D'Agostino R, Sullivan L, Elosua R, Wilson P, Ordovas J, Solanas P, Cordon F, Ramos R, Sala J, Masia R, Kannel WB: An adaptation of the Framingham coronary heart disease risk function to European Mediterranean areas. J Epidemiol Community Health 2003, 57:634-638.

16. Hense HW, Schulte H, Lowel H, Assmann G, Keil U: Framingham risk function overestimates risk of coronary heart disease in men and women from Germany - results from the MONICA Augsburg and the PROCAM cohorts. Eur Heart J 2003, 24:937-945.

17. Hobbs FD, Erhardt L: Acceptance of guideline recommendations and perceived implementation of coronary heart disease prevention among primary care physicians in five European countries: the Reassessing European Attitudes about Cardiovascular Treatment (REACT) survey. Fam Pract 2002, 19:596-604.

18. Thomsen TF, Jorgensen T, Ibsen H, Borch-Johnsen K: Assessment of coronary risk in general practice in relation to the use of guidelines: a survey in Denmark. Prev Med 200I, 33:300-304.

19. Kaufman DR, Kushniruk AW, Yale J-F, Patel V: Conceptual knowledge and decision strategies in relation to hypercholesterolemia and coronary heart disease. Int J Med Inf 1999, 55: I59- I 77.

20. Patel V, Arocha JF, Diermeier M, How J, Mottur-Pilson C: Cognitive psychological studies of representation and use of clinical practice guidelines. Int J Med Inf 200I, 63: I47-167.

\section{Pre-publication history}

The pre-publication history for this paper can be accessed here:

http://www.biomedcentral.com/1471-2296/5/3/prepub

Publish with Biomed Central and every scientist can read your work free of charge

"BioMed Central will be the most significant development for disseminating the results of biomedical research in our lifetime. "

Sir Paul Nurse, Cancer Research UK

Your research papers will be:

- available free of charge to the entire biomedical community

- peer reviewed and published immediately upon acceptance

- cited in PubMed and archived on PubMed Central

- yours - you keep the copyright

Submit your manuscript here:

http://www.biomedcentral.com/info/publishing_adv.asp
BiolMedcentral 